\title{
Semi-Analytical Solution Based on Strip Method for Buckling and Vibration of Isotropic Plate
}

\author{
Mohamed A. El-Sayad and Ahmed M. Farag \\ Department of Engineering Mathematics and Physics, Faculty of Engineering, Alexandria University, Alexandria, Egypt \\ Correspondence should be addressed to Mohamed A. El-Sayad; mel_sayad@hotmail.com
}

Received 31 August 2013; Accepted 29 October 2013

Academic Editor: Chein-Shan Liu

Copyright (c) 2013 M. A. El-Sayad and A. M. Farag. This is an open access article distributed under the Creative Commons Attribution License, which permits unrestricted use, distribution, and reproduction in any medium, provided the original work is properly cited.

\begin{abstract}
The present paper achieves a semianalytical solution for the buckling and vibration of isotropic rectangular plates. Two opposite edges of plate are simply supported and others are either free, simply supported, or clamped restrained against rotation. The general Levy type solution and strip technique are employed with transition matrix method to develop a semianalytical approach for analyzing the buckling and vibration of rectangular plates. The present analytical approach depends on reducing the strips number of the decomposed domain of plate without escaping the results accuracy. For this target, the transition matrix is expressed analytically as a series with sufficient truncation numbers. The effect of the uni-axial and bi-axial in-plane forces on the natural frequency parameters and mode shapes of restrained plate is studied. The critical buckling of rectangular plate under compressive in-plane forces is also examined. Analytical results of buckling loads and vibration frequencies are obtained for various types of boundary conditions. The influences of the aspect ratios, buckling forces, and coefficients of restraint on the buckling and vibration behavior of rectangular plates are investigated. The presented analytical results may serve as benchmark solutions for such plates. The convergence and efficiency of the present technique are demonstrated by several numerical examples compared with those available in the published literature. The results show fast convergence and stability in good agreement with compressions.
\end{abstract}

\section{Introduction}

The buckling problem of a thin rectangular elastic plate subjected to in-plane compressive forces is important in the aircraft and automotive industries. Kumar Panda and Ramachandra [1] offered a brief historical review on this subject. Due to the additional complexity of achieving the analytical solution of the plate problems under nonclassical boundary conditions, analysis for the effect of the in-plane force and buckling of the plate becomes difficult. Several methods, such as Rayleigh-Ritz, finite element, finite difference, and Fourier series method, are available. Singh and Dey [2] discussed the transverse vibration of rectangular plates subjected to in-plane forces by a difference based on variational approach. Finite strip transition matrix method (FSTM) was used by Farag and Ashour $[3,4]$ as a numerical technique depending on Runge-Kutta method to solve the vibration of rectangular plate as an initial value problem. Consequently, the method has been improved and applied successfully for several problems of stepped rectangular and applied forces [5]. The method was improved to analyze the rectangular plates subjected to a combination of flexural vibration and in-plane forces [6]. El sayad and Ghazy [7] applied Rayleigh-Ritz method for free vibration of Mindlin trapezoidal plates. Buckling and vibration of rectangular plates in the presence of in-plane forces have been investigated by Xiang and Wang [8] and Xiang and Wei [9], an exact solution for plates with two opposite edges simply supported. Paik and Thayamballi [10] used an analytical method to study the buckling strength characteristic of isotropic plates with two opposite edges simply supported and other edges elastically restrained. Akhavan et al. [11] established exact solutions for buckling analysis of rectangular Mindlin plates under in-plane loads resting on Pasternak elastic foundation. Gorman [12] used the superposition method to obtain the free vibration frequencies and buckling of in-plane loaded plates with rotational elastic edge supports. Kheirikhah et al. [13] analyzed the biaxial buckling of soft-core composite 


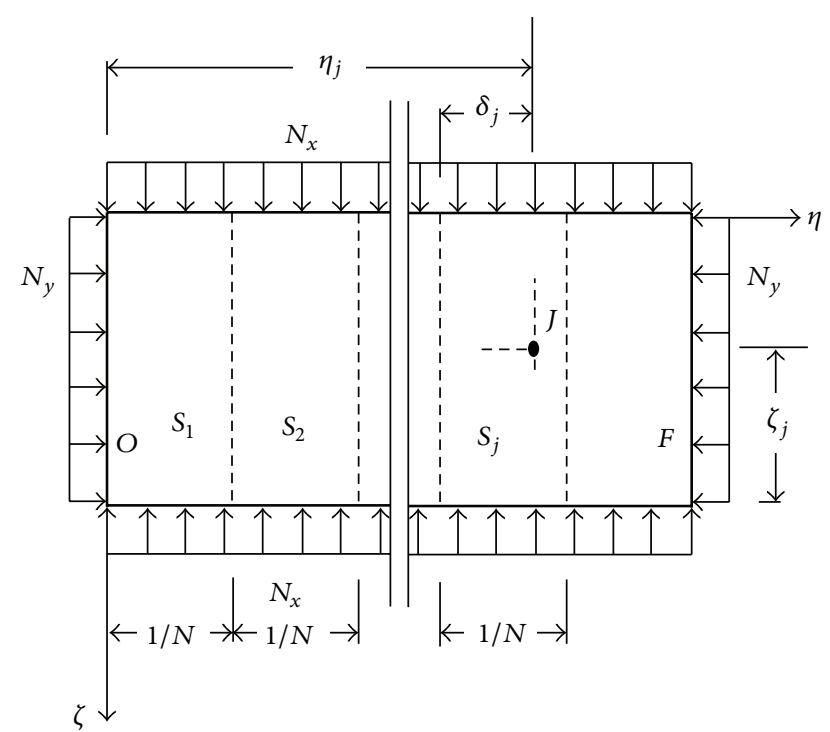

FIGURE 1: In-plane forces acting on isotropic rectangular plate.

sandwich plates using improved high-order theory. Bank and Yin [14] studied the uni-axial buckling of orthotropic plates with free and rotationally restrained unloaded edges. Lopatin and Morozov [15] studied the buckling of the SSCF rectangular orthotropic plate subjected to linearly varying in-plane loading. Wang [16] discussed buckling analysis of skew fiber-reinforced composite laminates based on firstorder shear deformation plate theory. Rodrigues et al. [17] applied the radial basis functions-differential quadrature collocation and a unified formulation to analyze the bending, vibration, and buckling of laminated plates according to Murakami's Zig-Zag theory. Buckling of thin skew fiberreinforced composite laminates was also studied by Wang [18]. Krishna Reddy and Palaninathan [19] investigated the case of buckling of laminated skew plates. Ovesy and Fazilati [20] analyzed the buckling and free vibration via finite strips of composite plates with cutout based on two different modeling approaches. Kaidas and Dickinson [21] used the numerical integration in a Rayleigh-Ritz to study the vibration and buckling calculations for rectangular plates subjected to complicated in-plane stress distributions. Mei and Yang [22] investigated the free vibrations of finite element plates subjected to complex middle-plane force systems. Zhang and Wang [23] studied elastoplastic buckling of thick rectangular plates by using the differential quadrature method. Gianetti et al. [24] and Diez et al. [25] used the Galerkin method to study the transverse vibrations of rectangular plates subject to in-plane and shear forces. Thai and Choi [26] applied an efficient and simple refined theory to analyze the buckling of functionally graded plates. Ibrahim et al. [27] studied buckling of composite thin walled beams by refined theory.

Recently, Farag and Ashour [3,4] expressed a semianalytical solution for vibrations of free stepped plate and buckling of plate subjected to in-plane forces. applying the transition matrix as a power matrix with exponential series which differs from one strip to another, producing a relatively length technique. In the present paper, the plate is divided into a limited number of equal strips. The transition matrix is expressed mathematically in explicit closed form to reduce the labor needed for crossing the proposed strips of plate. The plate strip is solved by a combination between the transition matrix and strip method. As can be seen, the present method directly gives a closed form expression for natural frequency of the investigated plate under a variation of boundary conditions at the initial and final ends of strips. Buckling and vibration of a rectangular plate subjected to normal in-plane forces are studied in the present paper. The studied plate is assumed to be rectangular, isotropic, and simply supported for the two opposite edges. Other edges are elastically restrained against rotation $E_{R}$. The simply supported edge $S$ and clamped edge $C$ are investigated as particular case of $E_{R}$. The achieved technique is applied to study the effect of uni-axial and biaxial in-plane forces on the critical buckling, natural frequency, and mode shape.

\section{Equation of Motion}

Regarding to the dimensionless partial differential equation of motion for vibrating plate subjected to in-plane forces $N_{x}$ and $N_{y}$ shown in Figure 1 is expressed as

$$
\begin{aligned}
\frac{\partial^{4} W}{\partial \zeta^{4}} & +2 \beta^{2} \frac{\partial^{4} W}{\partial \zeta^{2} \partial \eta^{2}}+\beta^{4} \frac{\partial^{4} W}{\partial \eta^{4}}-\frac{N_{x} a^{2}}{D} \frac{\partial^{2} W}{\partial \zeta^{2}} \\
& -\beta^{2} \frac{N_{y} a^{2}}{D} \frac{\partial^{2} W}{\partial \eta^{2}}+\frac{\bar{m} a^{4}}{D} \frac{\partial^{2} W}{\partial t^{2}}=0,
\end{aligned}
$$

where $\beta=a / b$ is the aspect ratio and $a, b$ are the dimensions of plate in $\zeta, \eta$ directions, respectively.

The other magnitudes $\bar{m}$ and $D$, respectively, are the plate mass per unit area and plate modulus of rigidity such that $D=E h^{3} /\left(12-\nu^{2}\right), E$ is the modulus of elasticity, $\nu$ is Poisson's ratio, and $h$ is the plate thickness. The plate is considered to be rectangular, isotropic, with edges simply supported $S$, clamped $C$, and restrained against rotation $E_{R}$. The transverse deflection $W$ is defined as

$$
W(\zeta, \eta, t)=\sum_{m=1}^{M} \Psi_{m}(\eta) \sin (m \pi \zeta) \sin \omega t
$$

where $\Psi_{m}(\eta)$ is unknown function to be determined for the boundary conditions at $\eta=0,1$.

Equation (2) is used to reduce the partial differential equation (1) into the following ordinary differential equation:

$$
\begin{aligned}
\Psi_{m}^{\prime \prime \prime \prime} & +\frac{1}{\beta^{2}}\left[-2 m^{2} \pi^{2}-\bar{N}_{y}\right] \Psi_{m}^{\prime \prime} \\
& +\frac{1}{\beta^{4}}\left[m^{2} \pi^{2} \bar{N}_{x}-\lambda_{m}^{2}+m^{4} \pi^{4}\right] \Psi_{m}=0
\end{aligned}
$$

where, $\lambda_{m}^{2}=\omega^{2} a^{4}(\bar{m} / D), \bar{N}_{x}=N_{x} a^{2} / D$, and $\bar{N}_{y}=N_{y} a^{2} / D$. 
Consequently, the plate equation of motion will be

$$
\begin{aligned}
\Psi_{m}^{\prime \prime \prime \prime} & \\
= & {\left[\frac{\left(-(m \pi)^{2} \bar{N}_{x}+\lambda_{m}^{2}-(m \pi)^{4}\right)}{\beta^{4}} 0\left(\frac{\bar{N}_{y}+2(m \pi)^{2}}{\beta^{2}}\right) 0\right] } \\
& \times\left\{\Psi_{m}\right\},
\end{aligned}
$$

where

$$
\left\{\Psi_{m}\right\}=\left[\begin{array}{c}
\Psi_{m} \\
\Psi_{m}^{\prime} \\
\Psi_{m}^{\prime \prime} \\
\Psi_{m}^{\prime \prime \prime}
\end{array}\right] .
$$

If the plate is divided into $N$ number of equal strips with constant dimensions and homogenous material, the general solution of the ordinary differential equation (4) can be expressed inside $j$ th strip at local distance $\delta_{j}$ from its initial edge as

$$
\left\{\Psi_{m}\right\}_{j}=\left[K_{m, 1 / N}\right]^{\mathrm{Q}=j}\left[K_{m, \delta_{j}}\right]\left\{\Psi_{m}\right\}_{0},
$$

where

$$
\left[K_{m, 1 / N}\right]=\left[\begin{array}{llll}
K_{11} & K_{12} & K_{13} & K_{14} \\
K_{21} & K_{22} & K_{23} & K_{24} \\
K_{31} & K_{32} & K_{33} & K_{34} \\
K_{41} & K_{42} & K_{43} & K_{44}
\end{array}\right]
$$

where the matrix elements $K_{i, j}, i=1,2,3,4$ and $j=$ $1,2,3,4$, are expressed in the Appendix.

In (6), the matrix power $Q$ is equal to the number $j$ of strips and matrix $\left[K_{m, \delta_{j}}\right]$ is obtained from $\left[K_{m, 1 / N}\right]$ by replacing $1 / N$ with $\delta_{j}$. Applying (6) for each nodal line at the end of each strip until the final end $F$ can be reached; one can obtain the final end vector as

$$
\left\{\Psi_{m}\right\}_{F}=\left[K_{m, 1 / N}\right]^{N}\left\{\Psi_{m}\right\}_{0} .
$$

Two characteristic equations for the plate vibration are obtained when the boundary conditions at $\eta=0,1$ are satisfied. The natural frequency or buckling parameters are the Eigen values of the characteristic matrix of these equations. The corresponding Eigen vectors create the mode shapes.

\section{Boundary and Initial Conditions}

Boundary conditions for edges elastically restrained against rotations $E_{R}$ at $(\eta=0,1)$ can be formulated as

$$
\begin{gathered}
\Psi_{m}=0, \quad \Psi_{m}^{\prime}=\mathfrak{R}_{0} \Psi_{m}^{\prime \prime} \quad \text { at } \eta=0, \\
\Psi_{m}=0, \quad \Psi_{m}^{\prime}=-\Re_{F} \Psi_{m}^{\prime \prime} \quad \text { at } \eta=1 .
\end{gathered}
$$

The magnitudes $\mathfrak{R}_{o}, \mathfrak{R}_{F}$ are restraint coefficients against rotation at $\eta=0,1$, respectively.

The initial vector $\left\{\Psi_{m}\right\}_{0}$ is expressed for various types of boundary conditions at $\eta=0$, namely, for edge elastically restrained against rotation, simply supported edge, clamped edge, respectively, such that

$$
\left\{\Psi_{m}\right\}_{0}=\left\{\begin{array}{c}
0 \\
b_{1} \mathfrak{R}_{0} \\
b_{1} \\
b_{2}
\end{array}\right\}, \quad\left\{\begin{array}{c}
0 \\
b_{1} \\
0 \\
b_{2}
\end{array}\right\}, \quad\left\{\begin{array}{c}
0 \\
0 \\
b_{1} \\
b_{2}
\end{array}\right\} .
$$

The magnitudes $b_{1}$ and $b_{2}$ are two arbitrary constants to be determined according to the proposed final boundary conditions at $\eta=0,1$.

\section{Buckling Natural Frequency and Mode Shape}

The final solution for buckling natural frequency parameters is obtained by applying the boundary conditions at the final edge of plate as in (8). Natural frequencies are the Eigen values of the characteristic equations:

$$
\left[\begin{array}{ll}
s_{11} & s_{12} \\
s_{21} & s_{22}
\end{array}\right]\left\{\begin{array}{l}
b_{1} \\
b_{2}
\end{array}\right\}=\left\{\begin{array}{l}
0 \\
0
\end{array}\right\} .
$$

Matrix $\left[A_{i j}\right], i=1,2, j=1,2$, matches the properties of plates and the coefficient of restraint against rotation. The mode shape vibration is given by the amplitude $W\left(\zeta_{j}, \eta_{j}\right)$ at point $N\left(\zeta_{j}, \eta_{j}\right)$ of plate such that

$$
\begin{array}{r}
\left\{W\left(\zeta_{j}, \eta_{j}\right)\right\}_{j}=\sum_{m=1}^{M} \sin \left(m \pi \zeta_{j}\right)\left[K_{m, \delta_{j}}\right]\left[K_{m, 1 / N}\right]^{j}\left\{\Psi_{m}\right\}_{0} \\
j=1,2,3, \ldots, N,
\end{array}
$$

where $\delta_{j}=\eta_{j}-j / N$. For each strip $S_{j}$, the local coordinate $\eta_{j}=j / N+\delta_{j}$ of point $N$ is referred to the first edge of $\operatorname{strip} \delta_{j}, j=1,2,3, \ldots, N$.

\section{Results and Discussions}

The reliability of present method is verified by means of many cases for plates under in-plane forces which are compared with the available exact values based on closed formulae [8]. Table 1 shows the convergence of the buckling load for full simply supported rectangular plate with aspect ratios $\beta=1,1.5,2$. The normalized uni-axial buckling load $\lambda_{\mathrm{cr}}=$ $-N_{x} a^{2} / D \pi^{2}$ is calculated using only four wide strips with truncation number $N$ varying from 2 to 6 . The results show fast convergence and good agreement with the exact values. In Table 2, the results of normalized uni-axial buckling load $\lambda_{\text {cr }}=-N_{x} a^{2} / D \pi^{2}$ for rectangular full simply supported plates SSSS under uni-axial load $N_{x}$ or $N_{y}$ are compared with those of available literature $[8,10]$. The results are calculated for aspect ratios $\beta$ varying from 0.15 to 2 . Also the results are obtained in Table 3 for SCSC rectangular plate subjected to uni-axial load $N_{x}$ or $N_{y}$ where the comparisons give excellent agreement. 
TABLE 1: Convergence of the buckling forces $\lambda_{\text {cr }}=-\left(N_{x} a^{2} / D \pi^{2}\right)$ of full simply supported rectangular isotropic plates under uni-axial load $N_{x}$.

\begin{tabular}{|c|c|c|c|c|c|c|}
\hline \multicolumn{7}{|c|}{ Critical buckling load $\lambda_{\text {cr }}$ due to $N, \beta$} \\
\hline$\beta$ & $N=2$ & $N=3$ & $N=4$ & $N=5$ & $N=6$ & Exact [8] \\
\hline 1.0 & 3.9997 & 4.0000 & 4.0000 & 4.0000 & 4.0000 & 4 \\
\hline 1.5 & 24.9971 & 24.9998 & 25.0000 & 25.0000 & 25.0000 & 25 \\
\hline 2.0 & 10.5615 & 10.5624 & 10.5625 & 10.5625 & 10.5625 & 10.5625 \\
\hline
\end{tabular}

TABLE 2: Comparisons for the buckling forces $\lambda_{\text {cr }}$ of rectangular isotropic plates SSSS under uni-axial load $N_{x}$; or $N_{x}$.

\begin{tabular}{|c|c|c|c|c|c|c|}
\hline \multirow{2}{*}{$\beta$} & \multicolumn{3}{|c|}{$\lambda_{\mathrm{cr}}=-\left(N_{x} a^{2} / D \pi^{2}\right)$} & \multicolumn{3}{|c|}{$\lambda_{\mathrm{cr}}=-\left(N_{y} a^{2} / D \pi^{2}\right)$} \\
\hline & Present & Exact [8] & {$[6]$} & Present & Exact $[8]$ & {$[6]$} \\
\hline 0.15 & 0.95816605 & & - & & & \\
\hline 0.25 & 1.128906247 & 1.128906252 & 1.128906247 & & & \\
\hline 0.35 & 1.260007167 & & & & & \\
\hline 0.50 & 1.56250000 & 1.56250000 & 1.562500000 & 4.09131879 & & 4.09131879 \\
\hline 0.65 & 2.023506256 & & & 4.281435537 & & \\
\hline 0.75 & 2.441406250 & 2.441406250 & 2.441406250 & 4.34021181 & 4.34027760 & 4.34021181 \\
\hline 0.85 & 2.967006237 & & & 4.106579447 & & \\
\hline 1.00 & 4.0000 & 4.00000000 & 3.999999990 & 4.00000006 & 4.00000000 & 4.00000006 \\
\hline 1.15 & 5.394006247 & & & 4.078643686 & & \\
\hline 1.25 & 6.566406250 & 6.566406250 & 6.566406250 & 4.20250002 & 4.20250000 & 4.20250002 \\
\hline 1.35 & 7.966506249 & & & 4.371196821 & & \\
\hline 1.50 & 10.56250000 & 10.56250000 & 10.56250000 & 4.69444443 & 4.69444442 & 4.69444443 \\
\hline 1.65 & 13.85700619 & & & 5.089809442 & & \\
\hline 1.75 & 16.50390623 & 16.50306239 & 16.50390623 & 5.38903626 & 5.38903061 & 5.38903626 \\
\hline 1.85 & 19.55850615 & & & 5.714684090 & & \\
\hline 2.00 & 25.00000000 & 25.00000000 & 24.99999999 & 6.25000001 & 6.25000000 & 6.25000001 \\
\hline
\end{tabular}

TABLE 3: Comparisons for the buckling forces $\lambda_{\text {cr }}$ of rectangular isotropic plates SCSC under uni-axial load $N_{x}$; or $N_{x}$.

\begin{tabular}{lcccc}
\hline$\beta$ & \multicolumn{2}{c}{$\lambda_{\text {cr }}=-\left(N_{x} a^{2} / D \pi^{2}\right)$} & \multicolumn{2}{c}{$\lambda_{\text {cr }}=-\left(N_{y} a^{2} / D \pi^{2}\right)$} \\
& Present & {$[6]$} & Present & {$[6]$} \\
\hline 0.50 & 1.922821352 & 1.922820900 & 4.047239747 & \\
0.75 & 4.002710767 & 4.002710767 & 5.553056186 & 5.52549351 \\
0.85 & 5.457252361 & & 5.910420828 & \\
1.00 & 8.604450855 & 8.604450855 & 6.74343791 & 6.74343791 \\
1.15 & 13.25680793 & & 7.854177784 & \\
1.25 & 17.41291859 & 17.41291859 & 8.72822985 & 8.72822985 \\
1.35 & 22.58349926 & & 9.700364123 & \\
1.50 & 32.59556551 & 32.59556551 & 11.3327751 & 11.3327751 \\
1.65 & 45.84690249 & & 13.16514162 & \\
1.75 & 56.80188160 & 56.80188160 & 14.4946584 & 14.4946584 \\
1.85 & 69.69626919 & & 15.90899653 & \\
2.00 & 93.16322000 & 93.16322000 & 18.1873798 & 18.1873798 \\
\hline
\end{tabular}

\section{Analytical Expression for Plate Natural Frequency}

Three cases of vibrations for plates subjected to in-plane forces and restrained boundary conditions are selected to show the analytical expressions of natural frequency. The plate has two opposite edges simply supported and the other edges are elastically restrained against rotation. The restrained coefficient is taken as 0.10 and the compression uni-axial loads are $N_{x}=N_{y}=-40,-30,-20$. In each case, the series expression of the natural frequency is obtained analytically by the computer algebraic solution Maple-13 as in (13)-(15). The obtained equations of frequency are plotted as shown in Figures 2, 3, and 4.

Case 1. If $N_{x}=N_{y}=-40$, the obtained frequency equation will be:

$$
\begin{aligned}
f(\lambda)= & -0.00133311724 \lambda^{2}+0.290110^{-14} \lambda^{8} \\
& -0.549510^{-19} \lambda^{10}+0.533310^{-24} \lambda^{12} \\
& -0.2920410^{-29} \lambda^{14}+0.9679910^{-35} \lambda^{16} \\
& -0.1957510^{-40} \lambda^{18}-0.687410^{-10} \lambda^{6} \\
& +0.59829910^{-6} \lambda^{4}+0.192710^{-46} \lambda^{20} \\
& +0.88210^{-53} \lambda^{22}-0.48710^{-58} \lambda^{24} \\
& +0.39710^{-64} \lambda^{2}+0.4610^{-70} \lambda^{28} \\
& -0.83810^{-76} \lambda^{30}+0.4710^{-83} \lambda^{32}
\end{aligned}
$$




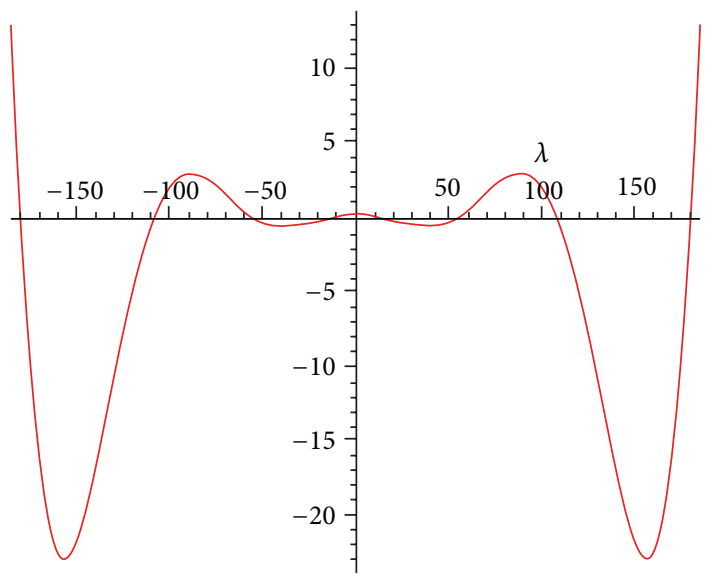

FIGURE 2: Graphical representation of frequency equation of Case 1.

$$
\begin{aligned}
& +0.128510^{-87} \lambda^{34}+0.15910^{-94} \lambda^{36} \\
& -0.283910^{-100} \lambda^{38}+0.138310^{-105} \lambda^{40} \\
& +0.22910^{-111} \lambda^{42}+0.142810^{-117} \lambda^{44} \\
& +0.398310^{-124} \lambda^{46}+0.291410^{-131} \lambda^{48} \\
& -0.509910^{-138} \lambda^{50}+0.2499=0 .
\end{aligned}
$$

The negative values of the solution are rejected and the positive solutions in some different modes are 14.354, 54.754, 108.348, and 179.813. Equation (13) is plotted in Figure 2 to show the possible values of natural frequency.

Case 2. If $N_{x}=N_{y}=-30$, the obtained frequency equation will be

$$
\begin{aligned}
f(\lambda)= & -0.00145 \lambda^{2}+0.6188210^{-6} \lambda^{4} \\
& -0.6988610^{-10} \lambda^{6}+0.2927910^{-14} \lambda^{8} \\
& -0.5526410^{-19} \lambda^{10}+0.5352810^{-24} \lambda^{12} \\
& -0.2928010^{-29} \lambda^{14}+0.9697910^{-35} \lambda^{16} \\
& -0.1958810^{-40} \lambda^{18}+0.192810^{-46} \lambda^{20} \\
& +0.8910^{-53} \lambda^{22}-0.48810^{-58} \lambda^{24} \\
& +0.39710^{-64} \lambda^{26}+0.46410^{-70} \lambda^{28} \\
& -0.83810^{-76} \lambda^{30}+0.510^{-83} \lambda^{32} \\
& +0.128510^{-87} \lambda^{34}+0.15910^{-94} \lambda^{36} \\
& -0.286510^{-100} \lambda^{38}+0.1378410^{-105} \lambda^{40} \\
& +0.228710^{-111} \lambda^{42}+0.142710^{-117} \lambda^{44}
\end{aligned}
$$

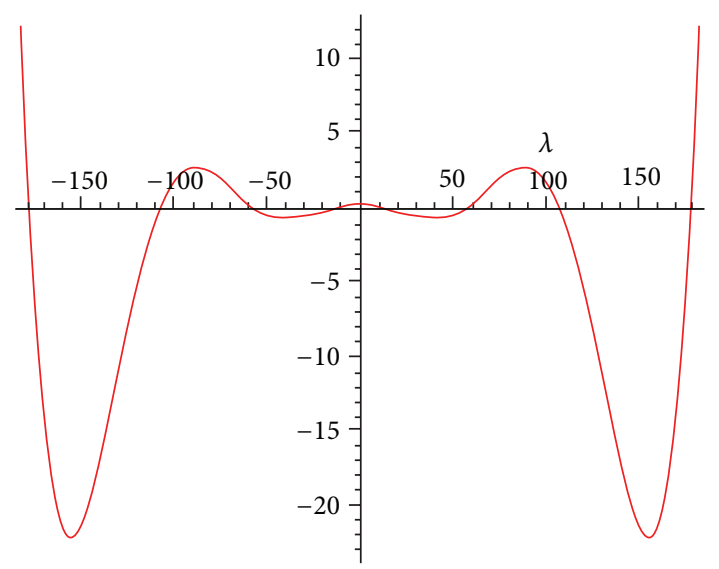

FIGURE 3: Graphical representation of frequency equation of Case 2.

$$
\begin{aligned}
& +0.398310^{-124} \lambda^{46}+0.291610^{-131} \lambda^{48} \\
& -0.509910^{-138} \lambda^{50}+0.3876=0 .
\end{aligned}
$$

The negative values of the solution are rejected and the positive solutions in some different modes are 17.457, 55.648, 108.803, and 180.088. Equation (14) is plotted in Figure 3 to show the possible values of natural frequency.

Case 3. If $N_{x}=N_{y}=-20$, the obtained frequency equation will be

$$
\begin{aligned}
f(\lambda)= & -0.00157 \lambda^{2}+0.6396810^{-6} \lambda^{4} \\
& -0.7104710^{-10} \lambda^{6}+0.2955210^{-14} \lambda^{8} \\
& -0.5558210^{-19} \lambda^{10}+0.537310^{-24} \lambda^{12} \\
& -0.2935610^{-29} \lambda^{14}-0.9714810^{-35} \lambda^{16} \\
& -0.1960910^{-40} \lambda^{18}+0.192610^{-46} \lambda^{20} \\
& +0.89210^{-53} \lambda^{22}-0.48910^{-58} \lambda^{24} \\
& +0.39310^{-64} \lambda^{26}+0.46510^{-70} \lambda^{28} \\
& -0.83910^{-76} \lambda^{30}+0.4710^{-83} \lambda^{32} \\
& +0.128310^{-87} \lambda^{34}+0.159110^{-94} \lambda^{36} \\
& -0.288910^{-100} \lambda^{38}+0.1373710^{-105} \lambda^{40} \\
& +0.2284010^{-111} \lambda^{42}+0.1426510^{-117} \lambda^{44} \\
& +0.3982010^{-124} \lambda^{46}+0.2916810^{-131} \lambda^{48} \\
& -0.5099310^{-138} \lambda^{50}+0.53688=0
\end{aligned}
$$

The negative values of the solution are rejected and the positive solutions in some different modes are 20.086, 56.528, 109.255, and 180.364. Equation (15) is plotted in Figure 4 to show the possible values of natural frequency. 


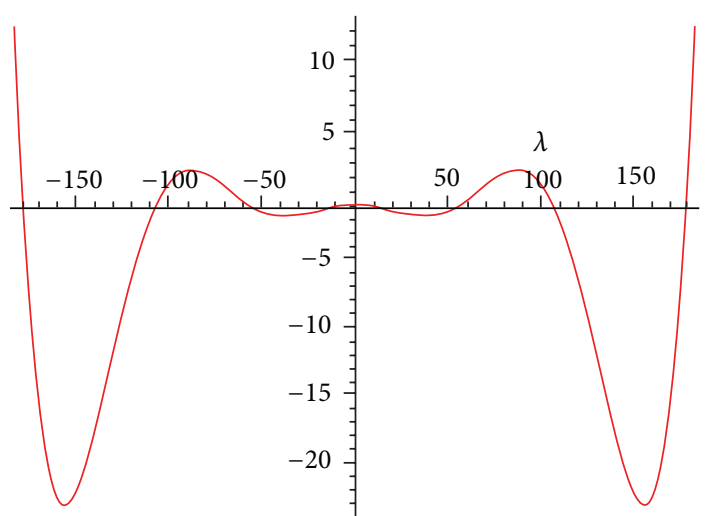

FIGURE 4: Graphical representation of frequency equation of Case 3.

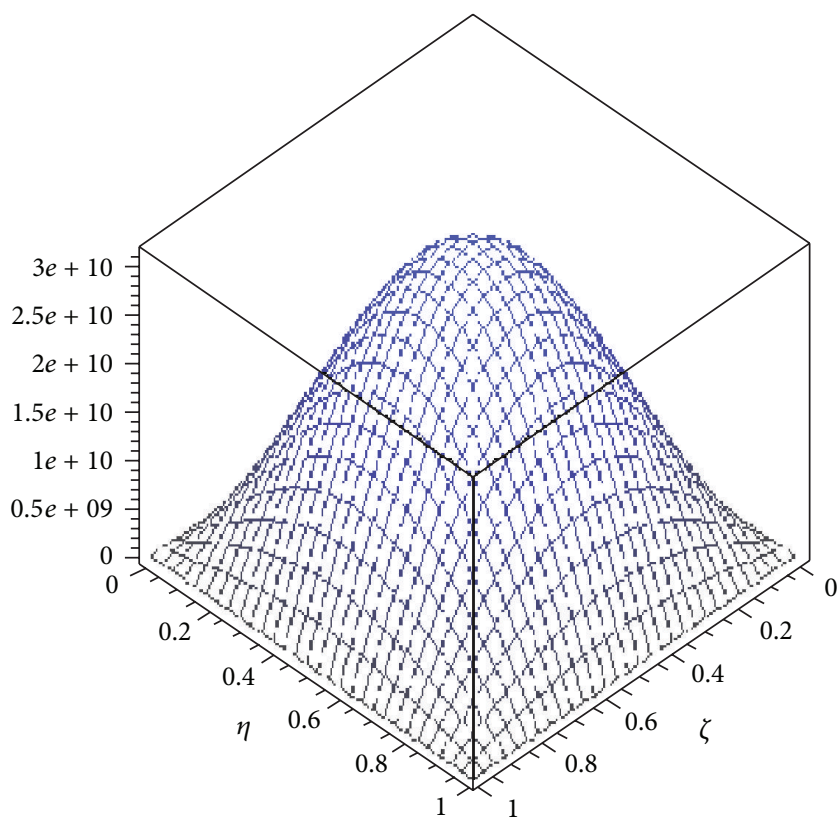

FIgURE 5: The first mode shape of the SSSS plate subjected to uniaxial in-plane force $\bar{N}_{x}=-20$.

\section{Analytical and Graphical Representation of the Mode Shape}

Mode shapes for five cases of SSSS and SCSC plate are obtained analytically as in (16)-(20). Also, the vibrating plate surfaces for these cases are represented graphically in 3D plots by means of Maple-13 as shown in Figures 5-9.

Case 1. In the first mode of the full simply supported square plate SSSS, when $N x=-20$ and $\lambda=13.8652$, the equation of the vibrating surface shape of the plate is

$$
\begin{aligned}
F(\xi, \eta)= & \sin (\pi \eta) \\
& \times\left(10^{11} \xi+0.50 \xi^{2}-0.164410^{12} \xi^{3}\right. \\
& \quad+0.8224 \xi^{4}+0.811710^{11} \xi^{5}+0.9470 \xi^{6}
\end{aligned}
$$

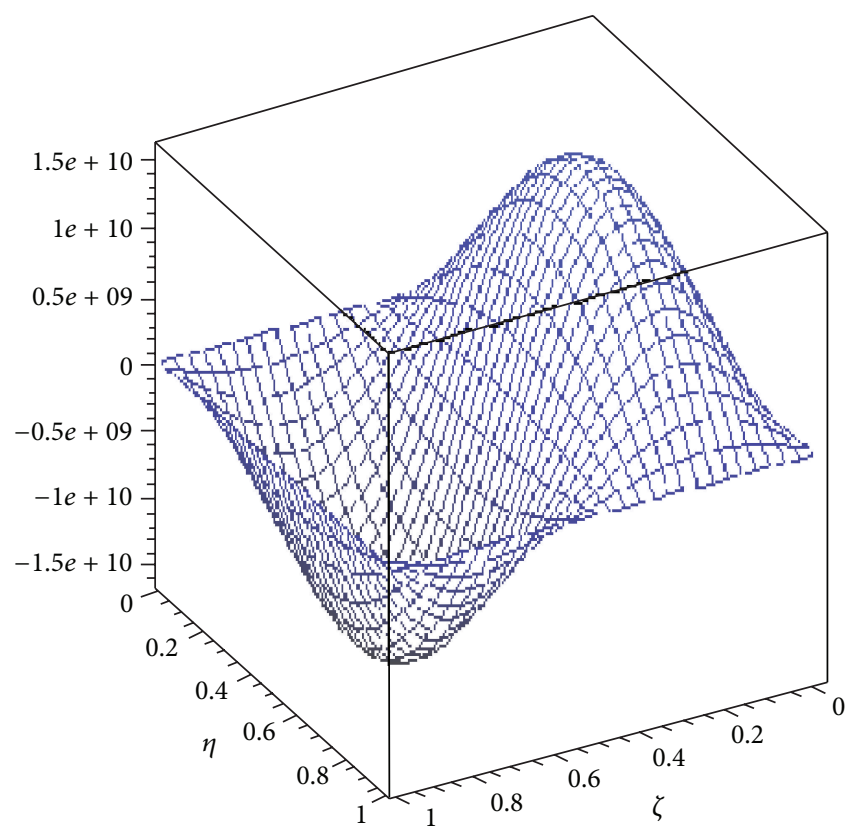

FIGURE 6: The first mode shape of the SSSS plate subjected to uniaxial in-plane force $\bar{N}_{x}=-40$.

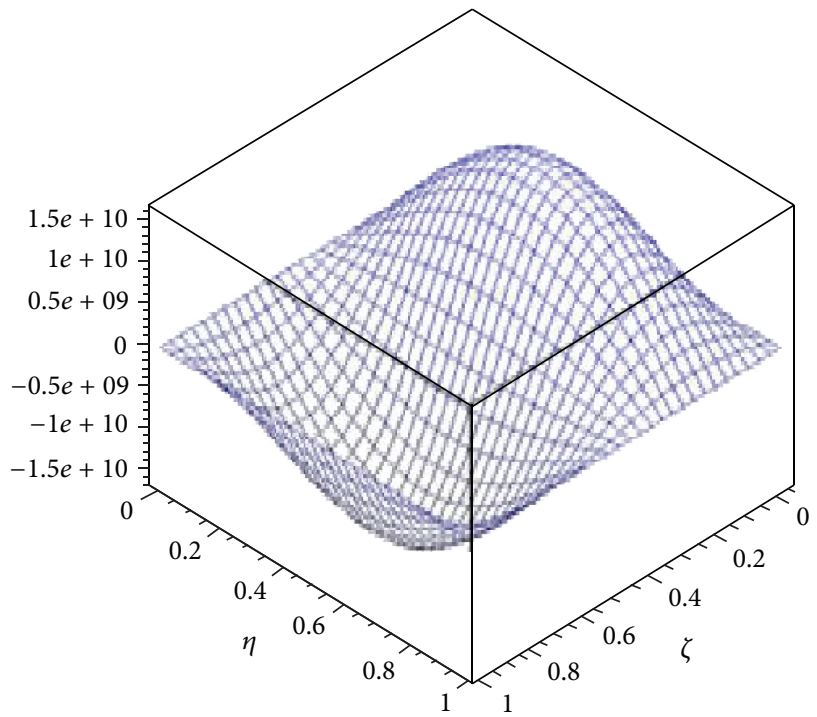

FIGURE 7: The second mode shape of the SSSS plate subjected to uniaxial in-plane force $\bar{N}_{x}=-20$.

$$
\begin{aligned}
& -0.190710^{11} \xi^{7}+0.4768 \xi^{8}+0.261410^{10} \xi^{9} \\
& +0.1595 \xi^{10}-0.234610^{9} \xi^{11}+0.0355 \xi^{12} \\
& +0.148410^{8} \xi^{13}+0.0057 \xi^{14}-697587 \xi^{15} \\
& +0.000715 \xi^{16}+25312.19 \xi^{17}+0.0000692 \xi^{18} \\
& \left.-730.469 \xi^{19}\right)=0 .
\end{aligned}
$$




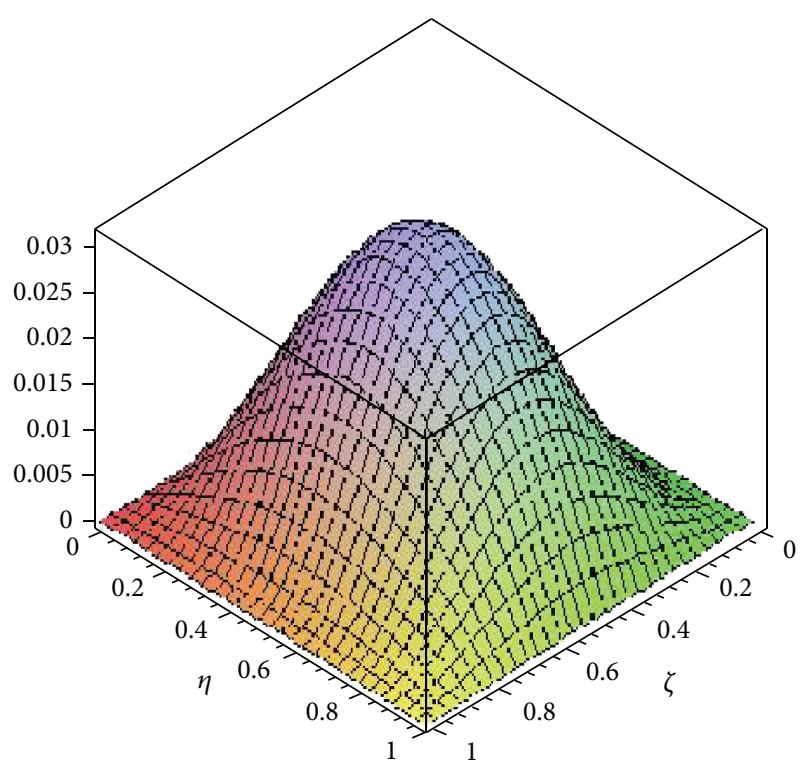

FIGURE 8: The first mode shape of the SCSC plate subjected to uniaxial in-plane force $\bar{N}_{x}=-40$.

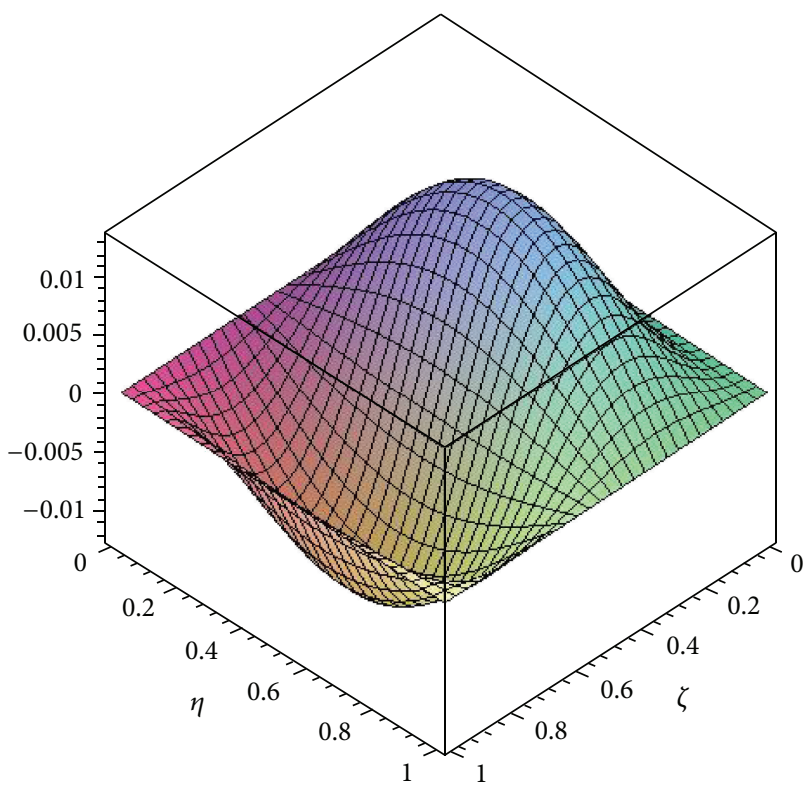

FIGURE 9: The second mode shape of the SCSC plate subjected to uni-axial in-plane force $\bar{N}_{x}=-40$.

The obtained equation is plotted to give the mode shape of the studied plate as shown in Figure 5.

Case 2. In the second mode of the full simply supported square plate SSSS, when $N x=-40$ and $\lambda=45.1201$, the equation of the vibrating surface shape of the plate is

$$
\begin{aligned}
F(\xi, \eta)= & \sin (\pi \eta) \\
& \times\left(10^{11} \xi+0.50 \xi^{2}-0.657110^{12} \xi^{3}\right.
\end{aligned}
$$

$$
\begin{aligned}
& +0.8224 \xi^{4}+0.129510^{11} \xi^{5} \\
& +3.78 \xi^{6}-0.121610^{13} \xi^{7} \\
& +2.47 \xi^{8}+0.6662 \xi^{9}+2.29 \xi^{10} \\
& -0.238810^{12} \xi^{11}+0.8291 \xi^{12} \\
& +0.603610^{11} \xi^{13}+0.3126 \xi^{14} \\
& -0.113310^{11} \xi^{15}+0.071 \xi^{16} \\
& +0.164310^{10} \xi^{17}+0.014410^{11} \xi^{18} \\
& \left.-0.189410^{9} \xi^{19}\right)=0 .
\end{aligned}
$$

The obtained equation is plotted to give the mode shape of the studied plate as shown in Figure 6.

Case 3. In the second mode of the full simply supported square plate SSSS, when $N x=-20$ and $\lambda=47.2595986$, the equation of the vibrating surface shape of the plate is

$$
\begin{aligned}
F(\xi, \eta)=\sin & (\pi \eta) \\
\times & \left(10^{11} \xi+0.50 \xi^{2}-0.657110^{12} \xi^{3}\right. \\
& +0.8224 \xi^{4}+0.129510^{13} \xi^{5}+3.78 \xi^{6} \\
& -0.121610^{13} \xi^{7}+2.47 \xi^{8}+0.666210^{12} \xi^{9} \\
& +2.29 \xi^{10}-0.238810^{12} \xi^{11}+0.8291 \xi^{12} \\
& +0.603610^{11} \xi^{13}+0.3126 \xi^{14}-0.113310^{11} \xi^{15} \\
& +0.071 \xi^{16}+0.164310^{10} \xi^{17}+0.01441 \xi^{18} \\
& \left.-0.189410^{9} \xi^{19}\right)=0 .
\end{aligned}
$$

The obtained equation is plotted to give the mode shape of the studied plate as shown in Figure 7.

Case 4. In the first mode of the SCSC, when $N x=-40$ and $\lambda$ $=21.09053660$, the equation of the vibrating surface shape of the plate is

$$
\begin{aligned}
F(\xi, \eta)= & \sin (\pi \eta) \\
\times & \left(0.50 \xi^{2}-1.03 \xi^{3}+0.8224 \xi^{4}\right. \\
& -1.02 \xi^{5}+1.57 \xi^{6}-1.394 \xi^{7} \\
& +0.917 \xi^{8}-0.632 \xi^{9}
\end{aligned}
$$




$$
\begin{aligned}
& +0.433 \xi^{10}-0.244 \xi^{11}+0.122 \xi^{12} \\
& -0.0583 \xi^{13}+0.0263 \xi^{14}-0.0113 \xi^{15} \\
& +0.00471 \xi^{16}-0.00156 \xi^{17} \\
& \left.+0.0005 \xi^{18}-0.000177 \xi^{19}\right)=0
\end{aligned}
$$

The obtained equation is plotted to give the mode shape of studied plate as shown in Figure 8.

Case 5. In the second mode of the SCSC, when $N x=-40$ and $\lambda=56.96696795$, the equation of the vibrating surface shape of the plate is

$$
\begin{aligned}
& F(\xi, \eta)=\sin (\pi \eta) \\
& \times( 0.50 \xi^{2}-1.39 \xi^{3}+0.8224 \xi^{4} \\
&-1.378 \xi^{5}+5.461 \xi^{6}-6.535 \xi^{7} \\
&+3.659 \xi^{8}-3.406 \xi^{9} \\
&+4.641 \xi^{10}-3.534 \xi^{11}+1.785 \xi^{12} \\
&-1.15 \xi^{13}+0.878 \xi^{14}-0.49 \xi^{15} \\
&+ 0.217 \xi^{16}-0.107 \xi^{17} \\
&\left.+0.0563 \xi^{18}-0.0248 \xi^{19}\right)=0 .
\end{aligned}
$$

The obtained equation is plotted to give the mode shape of the studied plate as shown in Figure 9.

\section{Conclusion}

Levy type solution and strip technique are employed with transition matrix method to develop a semianalytical approach for analyzing the buckling and vibration of rectangular plates. The reduction strips number for the decomposed domain of plate without escaping the results accuracy is the main idea of the present technique. For this target, the transition matrix is expressed analytically as a series with sufficient truncation numbers. The transition matrix is expressed mathematically in explicit closed form to reduce the labor needed for crossing the proposed strips of plate. The plate strip is solved by a combination between the transition matrix and strip method. As can be seen, the present method directly gives a closed form expression for natural frequency of the investigated plate under a variation of boundary conditions at the initial and final ends of strips. The effect of the uni-axial and biaxial in-plane forces on the natural frequency parameters and mode shapes of restrained plate is studied. The critical buckling of rectangular plate under compressive in-plane forces is examined. Analytical results of buckling loads and vibration frequencies are obtained for various types of boundary conditions. The influences of the aspect ratios, buckling forces, and coefficients of restraint on the buckling and vibration behavior of rectangular plates are investigated. The mode shape of the vibrating surface is specified in $3 \mathrm{D}$ graphs. The convergence stability of the present technique is examined and the obtained results are compared with those available in the published literature. The results show fast convergence and stability in good agreement with compressions.

\section{Appendix}

The matrix elements $K_{i, j}, i=1,2,3,4$, and $j=1,2,3,4$ are analytically obtained as

$$
\begin{aligned}
K_{11}= & 1+\frac{G_{41}}{24 N^{4}}+\frac{G_{43} G_{41}}{720 N^{6}}+\frac{G_{41}^{2}+G_{43}^{2} G_{41}}{40320 N^{8}}, \\
K_{12}= & \frac{1}{N}+\frac{G_{41}}{120 N^{5}}+\frac{G_{41} G_{43}}{5040 N^{7}}, \\
K_{13}= & \frac{1}{2 N^{2}}+\frac{G_{43}}{24 N^{4}}+\frac{G_{41}+G_{43}^{2}}{720 N^{6}} \\
& +\frac{G_{41} G_{43}+\left(G_{41}+G_{43}^{2}\right) G_{43}}{40320 N^{8}}, \\
K_{14}= & \frac{1}{6 N^{3}}+\frac{G_{43}}{120 N^{5}}+\frac{G_{41}+G_{43}^{2}}{5040 N^{7}}, \\
K_{21}= & \frac{G_{41}}{6 N^{3}}+\frac{G_{41} G_{43}}{120 N^{5}}+\frac{\left(G_{41}+G_{43}^{2}\right) G_{41}}{5040 N^{7}},
\end{aligned}
$$$$
K_{22}=1+\frac{G_{41}}{24 N^{4}}+\frac{G_{41} G_{43}}{720 N^{6}}+\frac{G_{41}^{2}+G_{43}^{2} G_{41}}{40320 N^{8}},
$$$$
K_{23}=\frac{1}{N}+\frac{G_{43}}{6 N^{3}}+\frac{G_{41}+G_{43}^{2}}{120 N^{5}}+\frac{G_{41} G_{43}+\left(G_{41}+G_{43}^{2}\right) G_{43}}{5040 N^{7}},
$$$$
K_{24}=\frac{1}{2 N^{2}}+\frac{G_{43}}{24 N^{4}}+\frac{G_{41}+G_{43}^{2}}{720 N^{6}}
$$$$
+\frac{G_{43} G_{41}+\left(G_{41}+G_{43}^{2}\right) G_{43}}{40320 N^{8}}
$$

$$
\begin{aligned}
K_{31}= & \frac{G_{41}}{2 N^{2}}+\frac{G_{41} G_{43}}{24 N^{4}}+\frac{G_{41}^{2}+G_{43}^{2} G_{41}}{720 N^{6}} \\
& +\frac{G_{43} G_{41}^{2}+\left(G_{41}+G_{43}^{2}\right) G_{41} G_{43}}{40320 N^{8}},
\end{aligned}
$$

$K_{32}=\frac{G_{41}}{6 N^{3}}+\frac{G_{41} G_{43}}{120 N^{5}}+\frac{G_{41}^{2}+G_{43}^{2} G_{41}}{5040 N^{7}}$,

$K_{33}=1+\frac{G_{43}}{2 N^{2}}+\frac{G_{41}+G_{43}^{2}}{24 N^{4}}+\frac{G_{41} G_{43}+\left(G_{41}+G_{43}^{2}\right) G_{43}}{720 N^{6}}$

$$
+\frac{G_{41} G_{43}^{2}+\left(G_{41}+G_{43}^{2}\right)^{2}}{40320 N^{6}}
$$

$K_{34}=\frac{1}{N}+\frac{G_{43}}{6 N^{3}}+\frac{G_{41}+G_{43}^{2}}{120 N^{5}}+\frac{G_{43} G_{41}+\left(G_{41}+G_{43}^{2}\right) G_{43}}{5040 N^{7}}$, 


$$
\begin{aligned}
K_{41}= & \frac{G_{41}}{N}+\frac{G_{41} G_{43}}{6 N^{3}}+\frac{\left(G_{41}+G_{43}^{2}\right) G_{41}}{120 N^{5}} \\
& +\frac{\left(G_{43} G_{41}+\left(G_{41}+G_{43}^{2}\right) G_{43}\right) G_{41}}{5040 N^{7}}, \\
K_{42}= & \frac{G_{41}}{2 N^{2}}+\frac{G_{41} G_{43}}{24 N^{4}}+\frac{\left(G_{41}+G_{43}^{2}\right) G_{41}}{720 N^{6}} \\
& +\frac{G_{43} G_{41}^{2}+\left(G_{41}+G_{43}^{2}\right) G_{43} G_{41}}{40320 N^{8}}, \\
K_{43}= & \frac{G_{43}}{N}+\frac{G_{41}+G_{43}^{2}}{6 N^{3}}+\frac{G_{41} G_{43}+\left(G_{41}+G_{43}^{2}\right) G_{43}}{120 N^{5}} \\
& +\frac{\left(G_{41}+G_{43}^{2}\right) G_{41}+\left(G_{41} G_{43}+\left(G_{41}+G_{43}^{2}\right) G_{43}\right) G_{43}}{5040 N^{7}}, \\
K_{44}= & 1+\frac{G_{43}}{2 N^{2}}+\frac{G_{41}+G_{43}^{2}}{24 N^{4}}+\frac{G_{41} G_{43}+\left(G_{41}+G_{43}^{2}\right) G_{43}}{720 N^{6}} \\
& +\frac{G_{41} G_{43}^{2}+\left(G_{41}+G_{43}^{2}\right)^{2}}{40320 N^{8}}, \\
G_{41}= & \frac{\left(-(m \pi)^{2} \bar{N}_{x}+\lambda_{m}^{2}-(m \pi)^{4}\right)}{\beta^{4}}, \\
G_{43}= & \left(\frac{\bar{N}_{y}+2(m \pi)^{2}}{\beta^{2}}\right) .
\end{aligned}
$$

\section{References}

[1] S. Kumar Panda and L. S. Ramachandra, "Buckling of rectangular plates with various boundary conditions loaded by non-uniform inplane loads," International Journal of Mechanical Sciences, vol. 52, no. 6, pp. 819-828, 2010.

[2] J. P. Singh and S. S. Dey, "Transverse vibration of rectangular plates subjected to in-plane forces by a difference based variational approach," International Journal of Mechanical Sciences, vol. 32, no. 7, pp. 591-599, 1990.

[3] A. M. Farag and A. S. Ashour, "Free vibration of orthotropic skew plates," Journal of Vibration and Acoustics, Transactions of the ASME, vol. 122, no. 3, pp. 313-317, 2000.

[4] A. Ashour and A. Farag, "A Combination between Laplace transform, strip Method and transition matrix for determination of dynamic response and damping effect of plates," International Journal of Acoustics and Vibration, vol. 5, no. 4, pp. 191-195, 2000.

[5] M. A. El sayad and A. M. Farag, "Numerical solution of vibrating double and triple-panel stepped thikness plate," Canadian Journal on Science and Engineering Mathematics, vol. 4, pp. 159168, 2012.

[6] A. M. Farag, "Buckling and flexural vibration of plates subjected to in-plane forces," International Review of Mechanical Engineering, vol. 3, no. 2, pp. 239-246, 2009.

[7] M. A. El sayad and S. Ghazy, "Rayleigh-ritz method for free vibration of mindlin trapezoidal plates," International Journal of Emerging Technology \& Advanced Engineering, vol. 2, no. 5, 2012.

[8] Y. Xiang and C. M. Wang, "Exact buckling and vibration solutions for stepped rectangular plates," Journal of Sound and Vibration, vol. 250, no. 3, pp. 503-517, 2002.

[9] Y. Xiang and G. W. Wei, "Exact solutions for buckling and vibration of stepped rectangular Mindlin plates," International Journal of Solids and Structures, vol. 41, no. 1, pp. 279-294, 2004.

[10] J. K. Paik and A. K. Thayamballi, "Buckling strength of steel plating with elastically restrained edges," Thin-Walled Structures, vol. 37, no. 1, pp. 27-55, 2000.

[11] H. Akhavan, S. Hosseini Hashemi, H. R. Damavandi Taher, A. Alibeigloo, and S. Vahabi, "Exact solutions for rectangular Mindlin plates under in-plane loads resting on Pasternak elastic foundation. Part I: buckling analysis," Computational Materials Science, vol. 44, no. 3, pp. 968-978, 2009.

[12] D. J. Gorman, "Free vibration and buckling of in-plane loaded plates with rotational elastic edge support," Journal of Sound and Vibration, vol. 229, no. 4, pp. 755-773, 2000.

[13] M. M. Kheirikhah, S. M. R. Khalili, and K. Malekzadeh Fard, "Biaxial buckling analysis of soft-core composite sandwich plates using improved high-order theory," European Journal of Mechanics A, vol. 31, pp. 54-66, 2012.

[14] L. C. Bank and J. Yin, "Buckling of orthotropic plates with free and rotationally restrained unloaded edges," Thin-Walled Structures, vol. 24, no. 1, pp. 83-96, 1996.

[15] A. V. Lopatin and E. V. Morozov, "Buckling of the SSCF rectangular orthotropic plate subjected to linearly varying inplane loading," Composite Structures, vol. 93, no. 7, pp. 19001909, 2011.

[16] S. Wang, "Buckling analysis of skew fiber-reinforced composite laminates based on first-order shear deformation plate theory," Composite Structures, vol. 37, no. 1, pp. 5-19, 1997.

[17] J. D. Rodrigues, C. M. C. Roque, A. J. M. Ferreira, M. Cinefra, and E. Carrera, "Radial basis functions-differential quadrature collocation and a unified formulation for bending, vibration and buckling analysis of laminated plates, according to Murakami's Zig-Zag theory," Computers and Structures, vol. 90-91, no. 1, pp. 107-115, 2012.

[18] S. Wang, "Buckling of thin skew fibre-reinforced composite laminates," Thin-Walled Structures, vol. 28, no. 1, pp. 21-41, 1997.

[19] A. R. Krishna Reddy and R. Palaninathan, "Buckling of laminated skew plates," Thin-Walled Structures, vol. 22, no. 4, pp. 241-259, 1995.

[20] H. R. Ovesy and J. Fazilati, "Buckling and free vibration finite strip analysis of composite plates with cutout based on two different modeling approaches," Composite Structures, vol. 94, no. 3, pp. 1250-1258, 2012.

[21] M. M. Kaidas and S. M. Dickinson, "Vibration and buckling calculations for rectangular plates subject to complicated inplane stress distributions by using numerical integration in a Rayleigh-Ritz analysis," Journal of Sound and Vibration, vol. 75, pp. 151-162, 1981.

[22] C. Mei and T. Y. Yang, "Free vibrations of finite element plates subjected to complex middle-plane force systems," Journal of Sound and Vibration, vol. 23, no. 2, pp. 145-156, 1972.

[23] W. Zhang and X. Wang, "Elastoplastic buckling analysis of thick rectangular plates by using the differential quadrature method," Computers \& Mathematics with Applications, vol. 61, no. 1, pp. 44-61, 2011. 
[24] C. E. Gianetti, L. Diez, and P. A. A. Laura, "Transverse vibrations of rectangular plates with elastically restrained edges and subject to in-plane shear forces," Journal of Sound and Vibration, vol. 54, pp. 409-417, 1977.

[25] L. Diez, C. E. Giánetti, and P. A. A. Laura, "A note on transverse vibrations of rectangular plates subject to in-plane normal and shear forces," Journal of Sound and Vibration, vol. 59, no. 4, pp. 503-509, 1978.

[26] H.-T. Thai and D.-H. Choi, "An efficient and simple refined theory for buckling analysis of functionally graded plates," Applied Mathematical Modelling, vol. 36, no. 3, pp. 1008-1022, 2012.

[27] S. M. Ibrahim, E. Carrera, M. Petrolo, and E. Zappino, "Buckling of composite thin walled beams by refined theory," Journal of Composite Structures, vol. 94, pp. 563-570, 2012. 


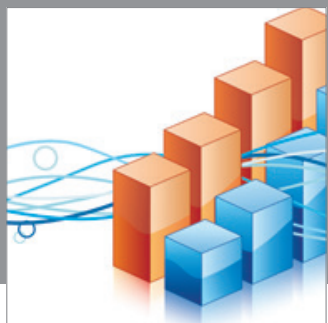

Advances in

Operations Research

mansans

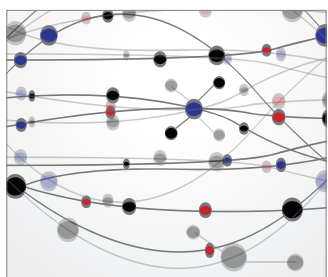

The Scientific World Journal
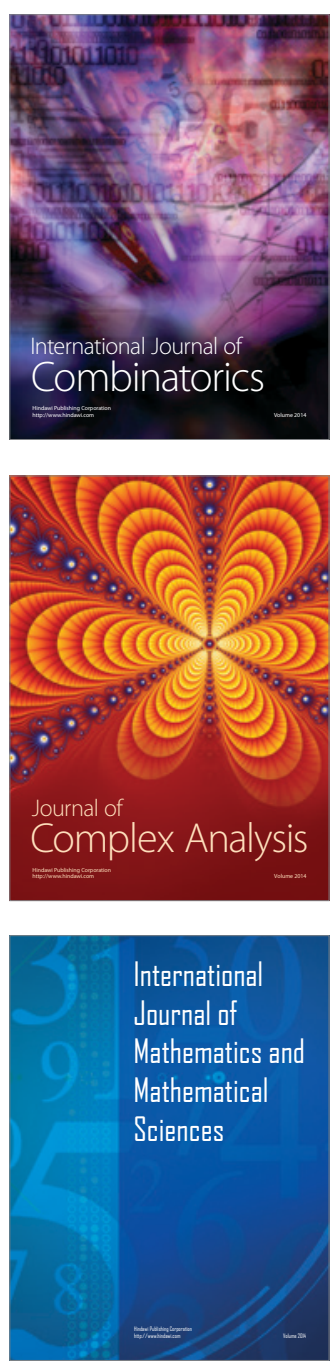
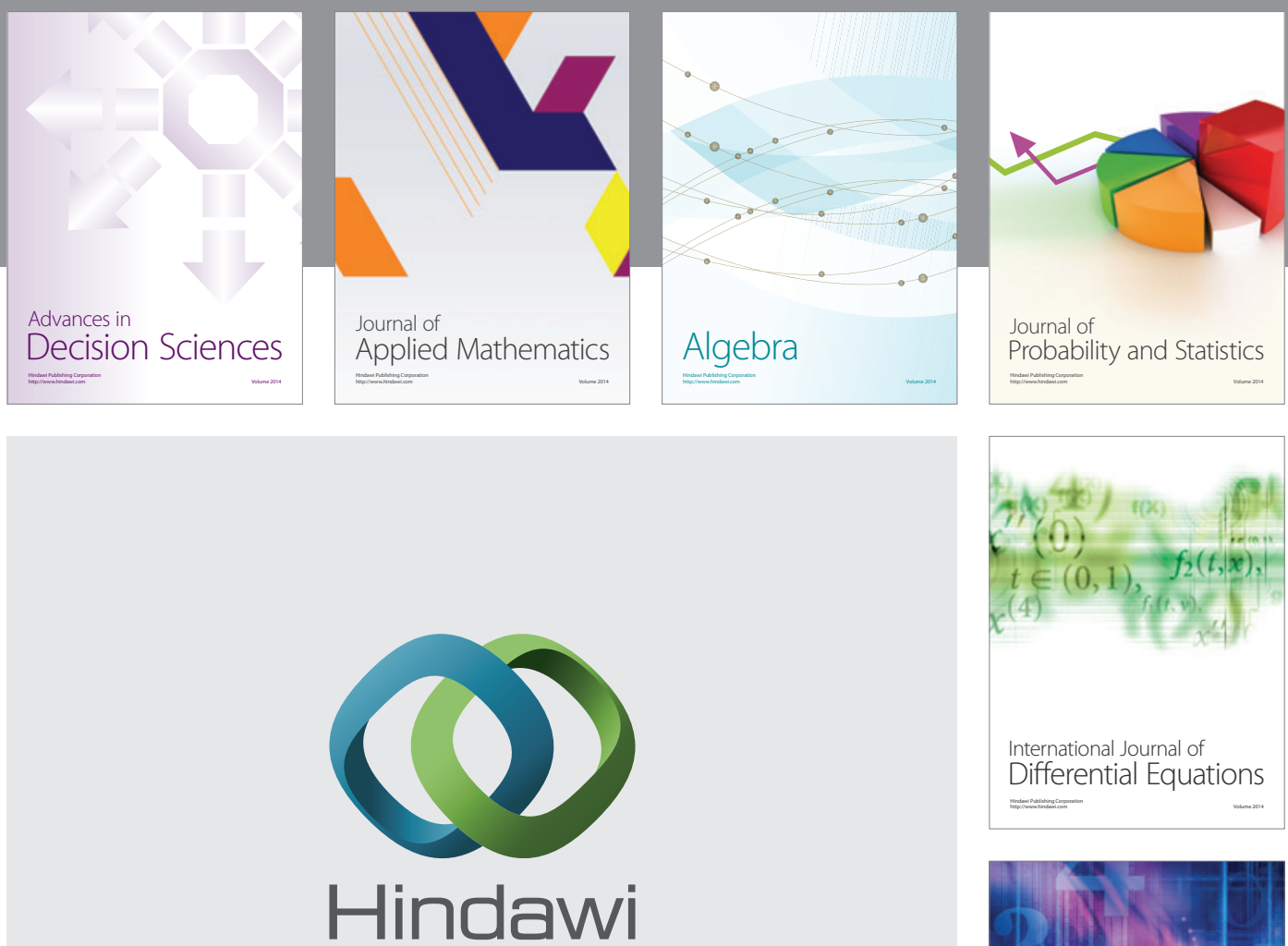

Submit your manuscripts at http://www.hindawi.com
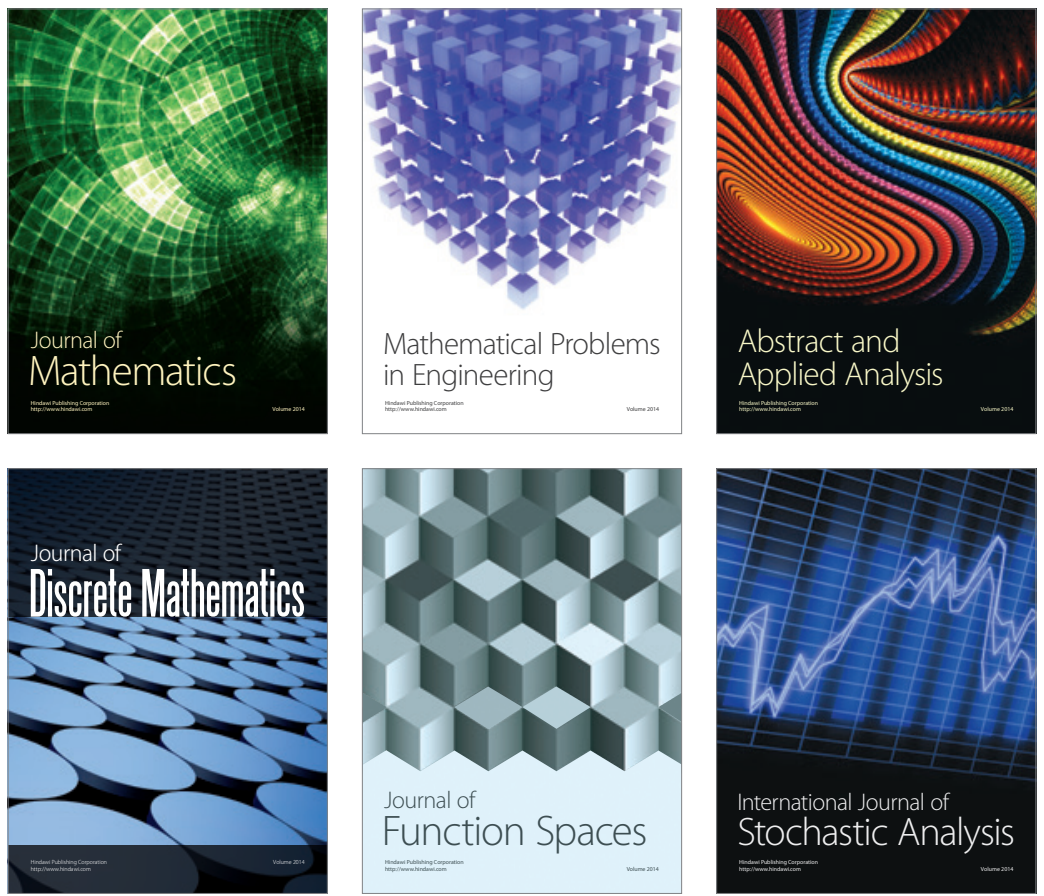

Journal of

Function Spaces

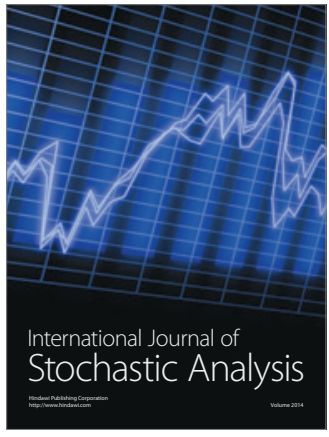

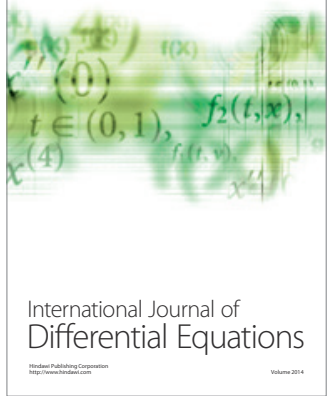
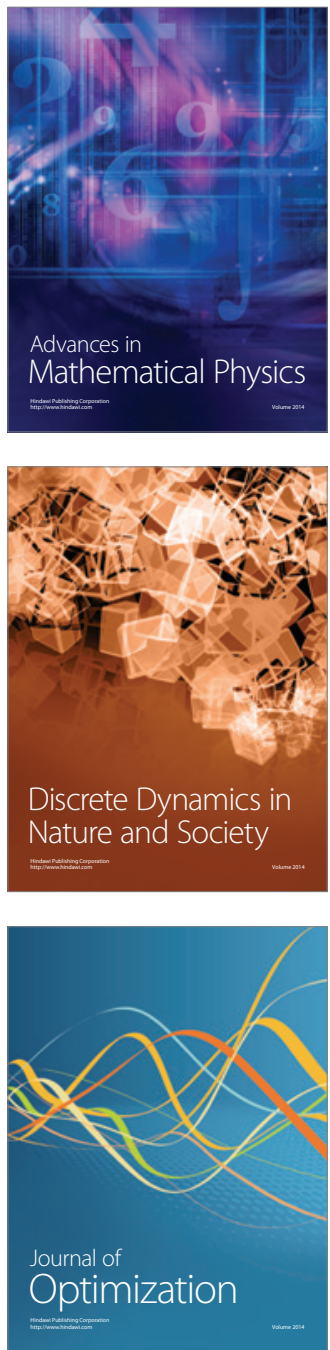